\title{
Genetic basis of hypercholesterolemia in adults
}

\author{
Seyedmohammad Saadatagah $\mathbb{D}^{1,4}$, Merin Jose ${ }^{1,4}$, Ozan Dikilitas $\mathbb{D}^{1}$, Lubna Alhalabi ${ }^{1}$, Alexandra A. Miller ${ }^{1}$, Xiao Fan ${ }^{1}$, Janet E. Olson ${ }^{2}$,
} David C. Kochan ${ }^{1}$, Maya Safarova ${ }^{1}$ and Iftikhar J. Kullo (iD ${ }^{1,3 凶}$

We investigated monogenic and polygenic causes of hypercholesterolemia in a population-based cohort, excluding secondary hypercholesterolemia, and using an established framework to identify pathogenic variants. We studied 1682 individuals (50.2 \pm 8.6 years, $41.3 \%$ males) from southeast Minnesota with primary hypercholesterolemia (low-density lipoprotein cholesterol (LDLC) $\geq 155 \mathrm{mg} / \mathrm{dl}$ in the absence of identifiable secondary causes). Familial hypercholesterolemia (FH) phenotype was defined as a Dutch Lipid Clinic Network (DLCN) score $\geq 6$. Participants underwent sequencing of $L D L R$, APOB, and PCSK9, and genotyping of 12 LDL-C-associated single-nucleotide variants to construct a polygenic score (PGS) for LDL-C. The presence of a pathogenic/likely pathogenic variant was considered monogenic etiology and a PGS $\geq 90$ th percentile was considered polygenic etiology. The mean LDL-C level was $187.3 \pm 32.3 \mathrm{mg} / \mathrm{dl}$ and phenotypic FH was present in $8.4 \%$ of the cohort. An identifiable genetic etiology was present in $17.1 \%$ individuals (monogenic in $1.5 \%$ and polygenic in 15.6\%). Phenotypic and genetic FH showed poor overlap. Only $26 \%$ of those who met the clinical criteria of $\mathrm{FH}$ had an identifiable genetic etiology and of those with an identifiable genetic etiology only $12.9 \%$ met clinical criteria for FH. Genetic factors explained $7.4 \%$ of the variance in LDL-C. In conclusion, in adults with primary hypercholesterolemia, $17.1 \%$ had an identifiable genetic etiology and the overlap between phenotypic and genetic FH was modest.

npj Genomic Medicine (2021)6:28; https://doi.org/10.1038/s41525-021-00190-z

\section{INTRODUCTION}

Hypercholesterolemia is a major risk factor for atherosclerotic cardiovascular disease (ASCVD) $)^{1,2}$. It is estimated that 95 million U.S. adults age 20 or older have an elevated cholesterol level with only half on lipid-lowering treatment ${ }^{3}$. Both genetic and lifestyle factors are known to predispose to hypercholesterolemia ${ }^{4,5}$. However, prior studies attempting to delineate the genetic basis of hypercholesterolemia included individuals already diagnosed (clinically) with familial hypercholesterolemia (FH) or those referred to lipid clinics. The reported prevalence of monogenic and polygenic etiology in such cohorts ranged from $1.7 \%-50 \%$ and $20 \%-30 \%$, respectively ${ }^{6-15}$. These estimates are affected by the referral bias inherent in such cohorts, inclusion of individuals with secondary forms of hypercholesterolemia, and variable application of guidelines to ascertain pathogenic variants in $\mathrm{FH}$ genes.

To address these limitations, we assessed the genetic basis of hypercholesterolemia in a cohort of individuals from the community who did not have a secondary cause for hypercholesterolemia. Pathogenic/likely pathogenic (P/LP) variants were identified by a molecular geneticist in a Central Laboratory Improvement Amendment (CLIA)-certified laboratory. We defined "phenotypic" FH, based on the Dutch Lipid Clinic Network (DLCN) criteria ${ }^{16}$ and "genetic" FH was defined as presence of a P/LP variant or elevated polygenic score (PGS) for low-density lipoprotein cholesterol (LDL-C). Additionally, we assessed the overlap between phenotypic and genetic forms of $\mathrm{FH}$.

\section{RESULTS}

\section{Participant characteristics and phenotypic FH}

Out of 38,258 Mayo Biobank participants, 2913 met study criteria and 1682 consented and participated in the study (Fig. 1). The mean $( \pm S D)$ age was $50.2 \pm 8.6$ years, $41.3 \%$ were males, $97.2 \%$ were non-Hispanic whites. The mean LDL-C level was $187.3 \pm$
$32.3 \mathrm{mg} / \mathrm{dl}, 590$ (35.1\%) participants had severe hypercholesterolemia (LDL-C $\geq 190 \mathrm{mg} / \mathrm{dl}$ ), 818 (48.6\%) had LDL-C $\geq 95$ th age-sexspecific percentile, and $372(22.1 \%)$ had LDL-C $\geq 99$ th age-sexspecific percentile. Phenotypic $\mathrm{FH}$, defined as $\mathrm{DLCN} \geq 6$, was present in 142 (8.4\%) (Table 1). Physical stigmata of FH were noted in only three patients, including two with tendon xanthomata and one with arcus cornealis.

\section{Monogenic and polygenic etiologies}

Sequencing of $L D L R, A P O B$ and PCSK9 identified 1300 variants of which 200 were rare and functional variants. Based on American College of Medical Genetics (ACMG) criteria, 25 participants (1.5\%) had P/LP variants. The number (percentage) of participants with variants in $L D L R, A P O B$, and PCSK9 were 18 (72\%), 6 (24\%), and 1 (4\%) respectively (Supplementary Table 2 ). The mean PGS in the study cohort was $0.97 \pm 0.21$ (Supplementary Fig. 2). Among participants without monogenic $\mathrm{FH}, 262$ (15.6\%) had polygenic hypercholesterolemia (PGS $\geq 1.16$, which corresponds to 90th percentile of PGS distribution in the UK-Whitehall II cohort). The mean LDL-C level in carriers of P/LP variants (monogenic FH) was $232.04 \pm 54.78 \mathrm{mg} / \mathrm{dl}$, significantly higher than those with PGS $>90$ th percentile (polygenic $\mathrm{FH}, 191.35 \pm 32.18 \mathrm{mg} / \mathrm{dl}$; $P$-value < 0.01 ), which in turn was higher than in those without these two conditions $(185.72 \pm 31.20 \mathrm{mg} / \mathrm{dl} ; P$-value $<0.01)$. (Fig. 2$)$ There were no significant differences between triglyceride and highdensity lipoprotein cholesterol levels between these three categories (Supplementary Fig. 3). Characteristics of clinical and genetic subgroups are provided in Supplementary Tables 3 and 4. Only $1.1 \%$ of the variance in LDL-C was explained by clinical and demographic factors, whereas $7 \%$ was explained by genetic factors (Supplementary Tables 6, 7).

${ }^{1}$ Department of Cardiovascular Medicine, Mayo Clinic, Rochester, MN, USA. ${ }^{2}$ Department of Health Sciences Research, Mayo Clinic, Rochester, MN, USA. ${ }^{3}$ Gonda Vascular Center, Mayo Clinic, Rochester, MN, USA. ${ }^{4}$ These authors contributed equally: Seyedmohammad Saadatagah, Merin Jose. ${ }^{\bowtie}$ email: kullo.iftikhar@mayo.edu 


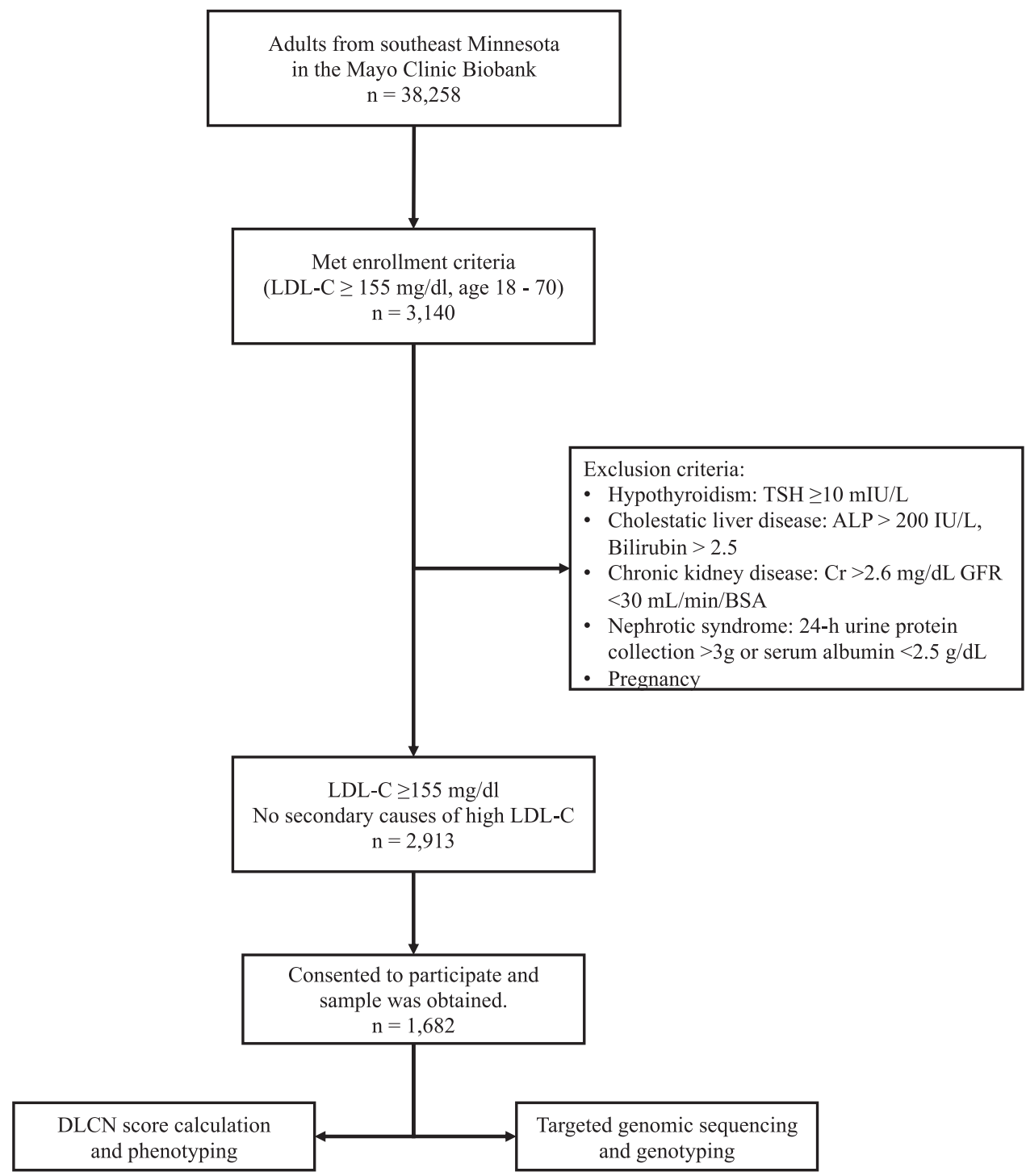

Fig. 1 Selection of the study cohort. ALP alkaline phosphatase, BSA body surface area, Cr creatinine, DLCN Dutch lipid clinic network, GFR glomerular filtration rate, LDL-C low-density lipoprotein cholesterol, TSH thyroid stimulating hormone.

\section{Overlap between phenotypic and genetic FH}

The overlap between those with a genetic etiology and those with phenotypic $\mathrm{FH}$ is summarized in Fig. 3. Among those with phenotypic $\mathrm{FH}$ (DLCN $\geq 6, n=142), 26.0 \%$ had a genetic etiology$7.0 \%$ monogenic and $19.0 \%$ polygenic. The distribution of monogenic $\mathrm{FH}$ cases across the spectrum of DLCN scores is depicted in Fig. 4. Among those with a genetic etiology $(n=287)$, $12.9 \%$ had phenotypic $\mathrm{FH}$; including $40.0 \%$ (10 out 25 ) with a monogenic etiology and $10.3 \%$ (27 out of 262 ) with a polygenic etiology, respectively. When considering only the LDL-C level, a monogenic etiology was present in $3.4 \%$ and $11.6 \%$ of those with $\mathrm{LDL}-\mathrm{C} \geq 190 \mathrm{mg} / \mathrm{dl} \quad(n=590)$ and $\mathrm{LDL}-\mathrm{C} \geq 250 \mathrm{mg} / \mathrm{dl} \quad(n=69)$, respectively. When participants were categorized based on their age-sex-specific LDL-C percentile, monogenic etiology of $\mathrm{FH}$ was present in $2.6 \%$ and $4.8 \%$ of those with LDL-C $\geq 95$ th $(n=818)$ and $\geq 99$ th $(n=372)$ percentile, respectively.

\section{DISCUSSION}

The main findings of our study of adults with primary hypercholesterolemia (i.e., LDL-C $\geq 155 \mathrm{mg} / \mathrm{dl}$ without an identifiable secondary cause) were: (1) a genetic etiology was present in
$17.1 \%$-monogenic in $1.5 \%$ and polygenic in $15.6 \%$; (2) $8.4 \%$ met DLCN criteria for $\mathrm{FH}$; (3) there was poor overlap between the presence of a genetic etiology and clinically diagnosed $\mathrm{FH}$. Our results suggest that genetic testing could be helpful for ascertaining $\mathrm{FH}$ cases and highlight the heterogeneity in the definitions of $\mathrm{FH}$.

The frequency of monogenic $\mathrm{FH}$ was $3.4 \%$ in individuals with LDL-C $\geq 190 \mathrm{mg} / \mathrm{dl}$, nearly double that (1.7\%) reported by Khera et al. $^{17}$ and higher than $2.5 \%$ reported by Abul-Husn et al. ${ }^{7}$. Several factors could account for differences in prevalence such as inclusion of patients with secondary hypercholesterolemia in the previous studies and differences in how P/LP variants were identified. When considering age-and sex-specific percentiles, monogenic etiology of $\mathrm{FH}$ was present in $2.6 \%$ and $4.8 \%$ of participants with LDL-C $\geq 95$ th and $\geq 99$ th percentile, respectively. The prevalence increased to $7.0 \%$ in individuals with phenotypic FH (DLCN score $\geq 6$ ). Our findings suggest that the yield of genetic testing in those with LDL-C $\geq 190 \mathrm{mg} / \mathrm{dl}$ is higher if secondary causes are ruled out and also if additional DLCN criteria are present. These data can inform clinicians regarding the expected yield of genetic testing in individuals with severe hypercholesterolemia. 
Conversely, more than half $(60.0 \%)$ of those with a monogenic etiology did not meet the DLCN criteria for $\mathrm{FH}$. In the study by Abul-Husn et al. ${ }^{7} 76.3 \%$ of individuals with a $\mathrm{P} / \mathrm{LP}$ variant in $\mathrm{FH}$ genes did not have phenotypic $\mathrm{FH}$. These observations indicate

Table 1. Participant characteristics $(n=1682)$.

\begin{tabular}{ll}
\hline Demographics & \\
\hline Age years, mean \pm SD & $50.2 \pm 8.6$ \\
Male, $n$ (\%) & $695(41.3 \%)$ \\
Whites, $n$ (\%) & $1635(97.2 \%)$ \\
Cardiovascular risk factors & \\
LDL-C mg/dl, mean \pm SD & $187.3 \pm 32.3$ \\
LDL-C $\geq 190$ mg/dl, $n$ (\%) & $590(35.1 \%)$ \\
LDL-C $\geq 95$ th percentile, $n$ (\%) & $818(48.6 \%)$ \\
LDL-C $\geq 99$ th percentile, $n$ (\%) & $372(22.1 \%)$ \\
Triglyceride mg/dl, mean \pm SD & $154.9 \pm 67.7$ \\
HDL-C mg/dl, mean \pm SD & $54.8 \pm 14.6$ \\
BMI kg/m ${ }^{2}$, mean \pm SD & $29.55 \pm 7.72$ \\
Diabetes, $n$ (\%) & $171(10.2 \%)$ \\
Hypertension, $n$ (\%) & $604(35.9 \%)$ \\
LDL-C on LLT, $n(\%)$ & $65(3.9 \%)$ \\
Premature ASCVD & \\
Personal history, $n$ (\%) & $307(18.3 \%)$ \\
Family history, $n$ (\%) & $287(17.1 \%)$ \\
Phenotypic FH & \\
Unlikely, $n$ (\%) & $926(55.0 \%)$ \\
Possible, $n$ (\%) & $614(36.6 \%)$ \\
Probable/definite, $n$ (\%) & $142(8.4 \%)$ \\
Genetic etiology & \\
Mongenic, $n(\%)$ & $262(15.6 \%)$ \\
MSCnic FH, $n(\%)$ & $25(1.5 \%)$ \\
\hline
\end{tabular}

ASCVD atherosclerotic cardiovascular disease, $B M I$ body mass index, $F H$ familial hypercholesterolemia, HDL-C high-density lipoprotein cholesterol, $L D L-C$ low-density lipoprotein cholesterol, $L L T$ lipid-lowering therapy. that clinical criteria are not sensitive in identifying individuals with monogenic $\mathrm{FH}$ and highlight the potential value of population genetic screening for FH case detection. Since presence of a P/LP variant is associated with increased coronary heart disease risk even after adjustment for LDL-C ${ }^{17}$, such knowledge will be useful in individualized care of affected individuals and also facilitate cascade testing of family members ${ }^{18}$.

The overall prevalence of a polygenic etiology (i.e., PGS $\geq 90$ th percentile of the general population) in our study was $15.6 \%$, $19.0 \%$ in those with clinical $\mathrm{FH}$, comparable with the prevalence of $20-30 \%$ in another cohort with clinical $\mathrm{FH}^{19}$. A polygenic etiology was thus 10-fold more common than monogenic etiology in individuals with phenotypic $\mathrm{FH}$. Increasing the number of single nucleotide variants (SNVs) in a PGS for LDL-C may categorize additional individuals as having a polygenic etiology. Our results suggest that a PGS for LDL-C may be useful in the clinical setting to establish the etiology of hypercholesterolemia including phenotypic $\mathrm{FH}$. Conversely, only $10.3 \%$ of those with polygenic etiology had a DLCN $\geq 6$.

In our study cohort of adults with primary hypercholesterolemia, variance in LDL-C explained by genetic factors (7.4\%) was greater than that explained by clinical/demographic variables (1.1\%). The latter is not unexpected since LDL-C is a heritable trait and heritable factors account for up to $60 \%$ of its inter-individual variance $^{20}$. In a substantial proportion, a genetic etiology was not identified, highlighting the need to identify additional etiologic factors for primary hypercholesterolemia. Such factors may include additional as yet uncharacterized monogenic/polygenic determinants, gene-gene and gene-environment interactions, and epigenetic effects ${ }^{21}$. In particular, in individuals with phenotypic $\mathrm{FH}$, no $\mathrm{P} / \mathrm{LP}$ variant in $L D L R, A P O B$ and $P C S K 9$ and a low polygenic score for LDL-C, a novel monogenic etiology may be present ${ }^{22}$. In such probands, family studies and further gene discovery may be warranted. In the present study, among those with phenotypic $\mathrm{FH}$, only $13(9.1 \%)$ individuals had low PGS (<20th percentile).

Our findings could have implications for clinical management of patients with $\mathrm{FH}$. Given the poor overlap between genetic and phenotypic $\mathrm{FH}$, genetic testing could be helpful for complete ascertainment of $\mathrm{FH}$ patients. Since more than half of the individuals with $\mathrm{P} / \mathrm{LP}$ variants did not meet clinical criteria for $\mathrm{FH}$, increased use of genetic testing as well as population scale genomic initiatives may be needed to identify individuals with
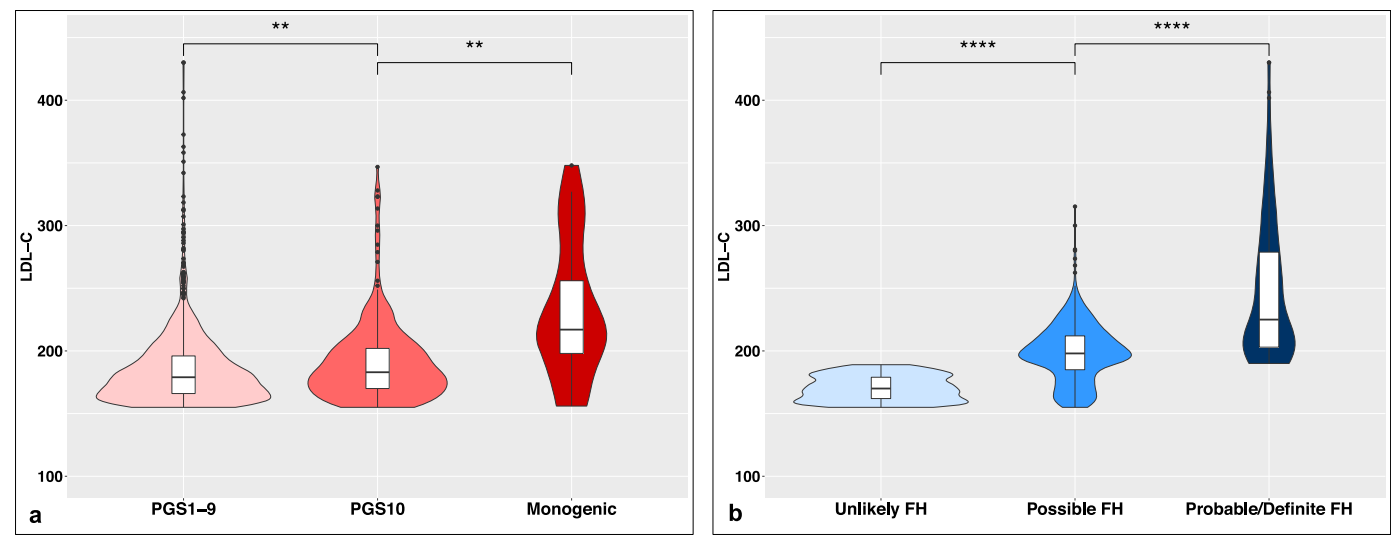

Fig. 2 Distribution of LDL-C levels in the study population. a Distribution of LDL-C levels based on genotype, and $\mathbf{b}$ based on phenotype. The boxplots are embedded in the density plots. The central line represents median, box limits represent upper and lower quartiles, the vertical lines represent $1.5 \times$ quartile range, and points represent outliers. In the left plot, PGS1-9 indicates those with the PGS in the 1 st to 9 th decile. PGS10 represents those with the PGS in the top decile (polygenic etiology of hypercholesterolemia) and Monogenic represents those with a P/LP variant in $L D L R, A P O B$ or PCSK9. On the right side, DLCN criteria are used for categorizing cases as Unlikely FH: DLCN $<3$, Possible $\mathrm{FH}: 3 \leq \mathrm{DLCN}<6$, and Probable/Definite FH: DLCN $\geq 6$. FH familial hypercholesterolemia, LDL-C low-density lipoprotein cholesterol, PGS polygenic score (number refers to PGS decile). $t$-test is used for comparison. ${ }^{*} P$-value $<0.05,{ }^{* *} P$-value $<0.01,{ }^{* * *} P$-value $<0.001,{ }^{* * * *} P$-value $<$ 0.0001 and $\mathrm{ns}=$ non-significant. 

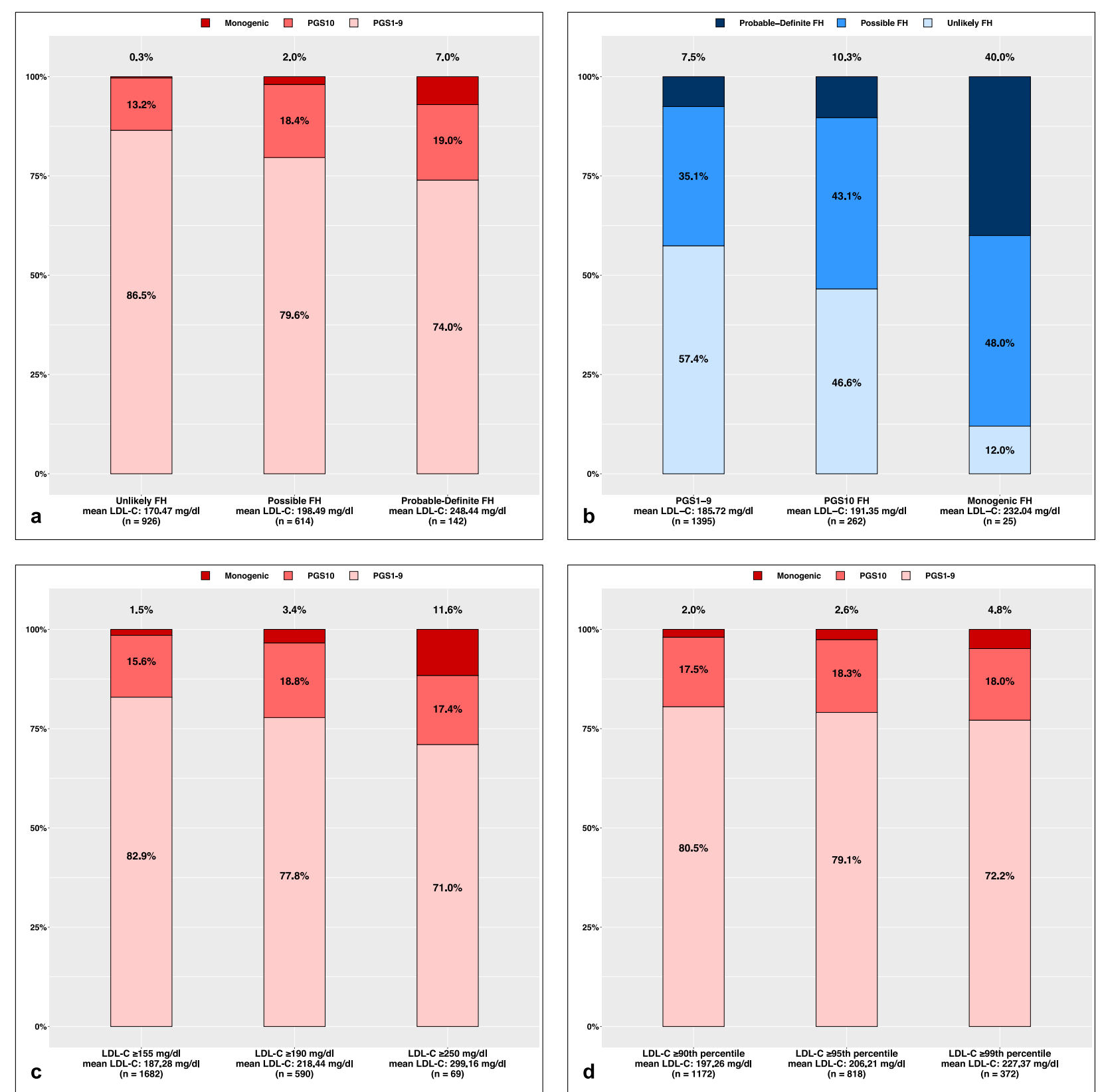

Fig. 3 Overlap of monogenic/polygenic FH and FH ascertained by clinical criteria. a Proportion of individuals in FH phenotypic categories who had an identifiable genetic etiology. b Proportion of individuals with polygenic or monogenic etiology for hypercholesterolemia who met the clinical criteria for FH. c Proportion of individuals in different LDL level who had an identifiable genetic etiology. d Proportion of individuals in different LDL percentiles who had an identifiable genetic etiology. FH familial hypercholesterolemia, LDL-C low-density lipoprotein cholesterol, PGS polygenic score. PGS1-9 indicates those with the PGS in the 1st to 9th decile. PGS10 represents those with the PGS in the top decile (polygenic etiology of hypercholesterolemia) and Monogenic represents those a P/LP variant in LDLR, $A P O B$ or PCSK9. DLCN criteria are used for the categorization of cases as Unlikely FH: DLCN <3, Possible FH: $3 \leq \mathrm{DLCN}<6$, and Probable/Definite FH: DLCN $\geq 6$.

monogenic FH who would otherwise go undetected. A substantial proportion of patients with clinical FH has polygenic hypercholesterolemia. Further research is needed to assess the utility of cascade testing in patients with clinical $\mathrm{FH}$ who do not have monogenic etiology ${ }^{23}$ and to identify new genetic etiologies in those with clinical $\mathrm{FH}$, no P/LP variant and a low PGS.

Pathogenic variants were identified by a molecular geneticist in a CLIA-certified laboratory, based on ACMG guidelines instead of solely relying on computational tools or databases. Phenotypic FH was ascertained using a validated algorithm for $\mathrm{FH}$ followed by manual review. The majority $(96.1 \%)$ of patients were treatment naïve at the time the highest LDL-C was recorded and in the remainder, we imputed pre-treatment $L D L-C$ based on the statin type and dose ${ }^{24}$. A limitation is lack of ethnic diversity in the study cohort. Participants were residents of Southeast Minnesota who participated in the Mayo Clinic Biobank and there may be a healthy participant and survivor bias. The $A P O B$ c.10580G $>$ A variant was present at high frequency in our study cohort despite absence of relatedness among carriers, suggesting a potential founder effect. The variant is enriched in populations of Amish descent ${ }^{25}$ and was also reported to be at a similar frequency in two other large cohorts ${ }^{7,26}$. At the time of design of this study, the 


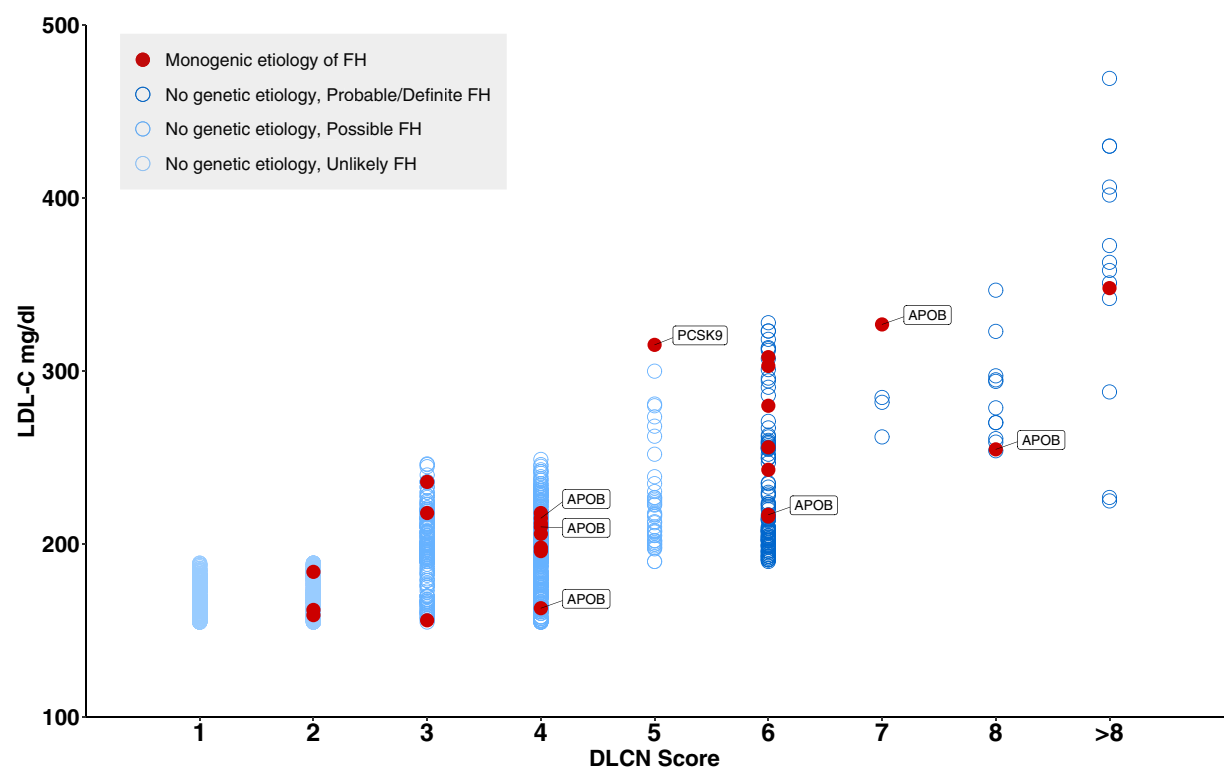

Fig. 4 Distribution of monogenic etiology across categories of DLCN score and corresponding LDL-C levels. Cases with P/LP APOB or PCSK9 variants are labeled and the remaining had P/LP LDLR variants. DLCN Dutch lipid clinic network, FH familial hypercholesterolemia, LDL-C lowdensity lipoprotein cholesterol.

available PGS for LDL-C included 12 LDL-C-associated SNVs; however, additional variants associated with LDL-C have been identified since ${ }^{27}$. A PGS incorporating a greater number of LDL-Cassociated variants would have explained a greater amount of variance in LDL-C and identified a greater proportion of our cohort as having a polygenic etiology. Nonetheless, we were able to infer that a polygenic etiology is present in at least $15.6 \%$ of cases with primary hypercholesterolemia and is much more frequent that monogenic etiology.

In conclusion, in adults with primary hypercholesterolemia (i.e., $\mathrm{LDL}-\mathrm{C} \geq 155 \mathrm{mg} / \mathrm{dl})$, a genetic etiology was present in $17.1 \%$ (monogenic in $1.5 \%$ and polygenic in $15.6 \%$ ) and genetic factors explained $7 \%$ of inter-individual variance in LDL-C. Given the modest overlap between the presence of a genetic etiology and phenotypic $\mathrm{FH}$, genetic testing could be helpful in identifying $\mathrm{FH}$ cases who might otherwise remain undetected.

\section{METHODS}

\section{Participant recruitment}

This study was approved by the Mayo Clinic Institutional Review Board. We identified Mayo Clinic Biobank ${ }^{28}$ participants from southeast Minnesota who were alive, aged between 18 and 70 years, and had hypercholesterolemia in the absence of a secondary cause. Hypercholesterolemia was defined as LDL-C level $\geq 155 \mathrm{mg} / \mathrm{dl}(\sim 4 \mathrm{mmol} / \mathrm{l})$ based on DLCN criteria ${ }^{16}$; secondary causes included hypothyroidism, cholestatic liver disease, severe kidney disease, nephrotic syndrome, and pregnancy detected within a 1-year window around the index date (first date of LDL$\mathrm{C} \geq 155 \mathrm{mg} / \mathrm{dl}$ ). Case selection and exclusion criteria have been previously reported $^{29}$ are also depicted in a flow chart (Fig. 1). Of 38,258 Mayo Biobank participants from southeast Minnesota, 1682 participants met inclusion criteria and consented to the study and were included in the final analysis.

\section{Targeted sequencing}

DNA of participant was sent to the Baylor College of Medicine Human Genome Sequencing Center, a CLIA-certified facility, for sequencing exons of the three FH genes (LDLR, APOB, PCSK9) and genotyping of 12 singlenucleotide variants (SNVs) associated with LDL-C (Supplementary Table 1). Details of sequencing methods and quality control metrics of the sequencing data have been previously described ${ }^{29,30}$. All three genes had $>99 \%$ of targeted bases sequenced to redundant coverage of $\geq 20$. Sequence data were analyzed by the Mercury 3.4 pipeline $^{31}$ (additional details of variant calling are described in Supplementary Method). The data from Illumina HiSeq were converted from bcl file to FASTQ file by Illumina bcl2fastq 1.8.3 software, and mapped to the hg19 human genome reference by the Burrows-Wheeler Aligner ${ }^{32}$. Both SNVs and copy number variants (CNV) were called using Atlas-SNP and Atlas-indel, respectively (additional details of CNV detection are provided in Supplementary Method). Variants passing quality control were mapped to gene loci using SeattleSeqAnnotation138. Principal component analysis of genetic ancestry and qualitative comparison to self-reported ancestry were performed as a part of quality control analyses (details provided in the Supplementary Method and Supplementary Fig. 1). The self-reported ancestry and genetically determined ancestry matched in our study participants.

\section{Monogenic etiology: identifying P/LP Variants}

The final variant annotation was based on ACMG/Association of Medical Pathology (ACMG/AMP) criteria ${ }^{30,33}$. Variants in $L D L R, A P O B$ and PCSK9 meeting the following criteria were identified using InterVar ${ }^{34}$ : (a) functional variants - missense, stop-gain, etc. (b) frequency of $<0.1 \%$ in ExAC ${ }^{35}$ or gnom $A D^{36}$, and (c) satisfying criteria for pathogenicity listed in the ACMG guideline ${ }^{33}$. Additional review included: (a) databases, i.e., ClinVar ${ }^{37}$, Leiden Open Variation Database ${ }^{38}$ and Human Gene Mutation Database $^{39}$, (b) relevant literature, and (c) clinical features reported in the electronic health record (EHR).

\section{Polygenic etiology: polygenic score for LDL-C}

A previously validated $12-S N V$ PGS $^{19}$ was used to measure the polygenic component of elevated LDL-C level (Supplementary Table 1). For each individual, a PGS for LDL-C-was calculated using a weighted sum of the effect alleles at the 12 SNVs. The weights used were the corresponding per-effect allele beta coefficients reported in a genome wide association study meta-analysis ${ }^{19,29}$. The PGS calculated for each individual was compared to the distribution of the score in the Whitehall II cohort ${ }^{19}$ and a $P G S \geq 90$ th percentile (i.e., $P G S \geq 1.16$ ) was considered as polygenic hypercholesterolemia, as in the derivation cohort, the mean LDL-C level of those with PGS $\geq 90$ th percentile was $190 \mathrm{mg} / \mathrm{dl}$, which is the threshold for defining severe hypercholesterolemia ${ }^{19}$.

\section{Phenotypic FH}

The previously validated SEARCH algorithm ${ }^{40}$ was used to extract DLCN scores from the EHR. Participants were classified as "definite", "probable", 
"possible" and "unlikely" FH based on a DLCN score $>8,6-8,3-5$, and $<3$, respectively. Phenotypic FH was defined as DLCN score $\geq 6$. The EHRs of individuals with DLCN score $\geq 6$ and those with monogenic FH were manually reviewed to confirm the presence of clinical criteria for FH. For individuals reporting statin use at the index date, untreated LDL-C level was imputed based on the type and dose of the statin, ascertained from the EHR, using a dynamic coefficient ${ }^{24}$. Premature ASCVD was considered as coronary heart disease, cerebrovascular disease, or peripheral artery disease in males before age 55 years and females before age 65 years ${ }^{40}$. In addition to using actual LDL-C level, we also estimated sex-, age-specific LDL-C percentile based on recent data derived from a white population ${ }^{41}$.

\section{Statistical analysis}

Categorical variables are presented as counts (percentages), and continuous variables as mean \pm SD. Group comparison for categorical and numerical variables was done using chi-square, $t$-test, and analysis of variances test, as appropriate. The amount of inter-individual variance in LDL-C explained by genetic factors and clinical and demographic factors (age, sex, race, body mass index, diabetes and family history of hypercholesterolemia) was estimated using a multivariable linear regression analysis. The details of regression models are provided in the supplementary material. Statistical analyses were performed using RStudio version 1.2.5033 (RStudio, Inc., Boston, MA). All tests were two-sided, and Pvalues $<0.05$ were considered statistically significant.

\section{Reporting summary}

Further information on experimental design is available in the Nature Research Reporting Summary linked to this paper.

\section{DATA AVAILABILITY}

The dataset generated and/or analyzed during the current study are available from the corresponding author on reasonable request. All eMERGE III data are available on dbGaP using "phs001616.v2.p2" accession code. More details could be found using the following link https://www.ncbi.nlm.nih.gov/projects/gap/cgi-bin/study. cgi?study_id=phs001616.v2.p2.

Received: 27 October 2020; Accepted: 26 February 2021; Published online: 14 April 2021

\section{REFERENCES}

1. Yusuf, P. S. et al. Effect of potentially modifiable risk factors associated with myocardial infarction in 52 countries (the INTERHEART study): Case-control study. Lancet 364, 937-952 (2004).

2. Virani, S. S. et al. Heart disease and stroke statistics - 2020 update: A report from the American Heart Association. Circulation 14, E139-E596 (2020).

3. Mercado, C. et al. Prevalence of cholesterol treatment eligibility and medication use among adults-United States, 2005-2012. Morb. Mortal. Wkly. Rep. 64, 1305-1311 (2015).

4. Heller, D. A., de Faire, U., Pedersen, N. L., Dahlen, G. \& McClearn, G. E. Genetic and environmental influences on serum lipid levels in twins. N. Engl. J. Med. 328, 1150-1156 (1993).

5. Anderson, J. T. et al. Scoring systems for evaluating dietary pattern effect on serum cholesterol. Prev. Med. (Balt.). 8, 525-537 (1979).

6. Khera, A. V. et al. Genetic risk, adherence to a healthy lifestyle, and coronary disease. N. Engl. J. Med. 375, 2349-2358 (2016).

7. Abul-Husn, N. S. et al. Genetic identification of familial hypercholesterolemia within a single U.S. Health care system. Science (80-.). 354, aaf7000 (2016).

8. Wang, J. et al. Polygenic versus monogenic causes of hypercholesterolemia ascertained clinically. Arterioscler. Thromb. Vasc. Biol. 36, 2439-2445 (2016).

9. Balder, J. W. et al. Genetics, lifestyle, and low-density lipoprotein cholesterol in young and apparently healthy women. Circulation 137, 820-831 (2018).

10. Ahmad, Z., Adams-Huet, B., Chen, C. \& Garg, A. Low prevalence of mutations in known loci for autosomal dominant hypercholesterolemia in a multiethnic patient cohort. Circ. Cardiovasc. Genet. 5, 666-675 (2012).

11. Humphries, S. E. et al. Genetic causes of familial hypercholesterolaemia in patients in the UK: relation to plasma lipid levels and coronary heart disease risk. J. Med. Genet. 43, 943-949 (2006).

12. Futema, M. et al. Analysis of the frequency and spectrum of mutations recognised to cause familial hypercholesterolaemia in routine clinical practice in a UK specialist hospital lipid clinic. Atherosclerosis 229, 161-168 (2013).
13. Taylor, A. et al. Mutation detection rate and spectrum in familial hypercholesterolaemia patients in the UK pilot cascade project. Clin. Genet. 77, 572-580 (2010).

14. Graham, C. A. et al. Genetic screening protocol for familial hypercholesterolemia which includes splicing defects gives an improved mutation detection rate. Atherosclerosis 182, 331-340 (2005).

15. Trinder, M. et al. Risk of premature atherosclerotic disease in patients with monogenic versus polygenic familial hypercholesterolemia. J. Am. Coll. Cardiol. 74, 512-522 (2019).

16. Nordestgaard, B. G. et al. Familial hypercholesterolaemia is underdiagnosed and undertreated in the general population: Guidance for clinicians to prevent coronary heart disease. Eur. Heart J. 34, 3478-3490 (2013).

17. Khera, A. V. et al. Diagnostic yield and clinical utility of sequencing familial hypercholesterolemia genes in patients with severe hypercholesterolemia. J. Am. Coll. Cardiol. 67, 2578-2589 (2016).

18. Patel, A. P. et al. Association of rare pathogenic DNA variants for familial hypercholesterolemia, hereditary breast and ovarian cancer syndrome, and lynch syndrome with disease risk in adults according to family history. JAMA Netw. Open 3, e203959 (2020).

19. Talmud, P. J. et al. Use of low-density lipoprotein cholesterol gene score to distinguish patients with polygenic and monogenic familial hypercholesterolaemia: a case-control study. Lancet 381, 1293-1301 (2013).

20. Walker, C. G. \& Jebb, S. A. Gene-diet interactions on lipid levels: current knowledge in the era of genome-wide association studies. Curr. Nutr. Rep. 1, 123-131 (2012).

21. Guay, S. P., Brisson, D., Lamarche, B., Gaudet, D. \& Bouchar, L. Epipolymorphisms within lipoprotein genes contribute independently to plasma lipid levels in familial hypercholesterolemia. Epigenetics 9, 718-729 (2014).

22. Futema, M. et al. Refinement of variant selection for the LDL cholesterol genetic risk score in the diagnosis of the polygenic form of clinical familial hypercholesterolemia and replication in samples from 6 countries. Clin. Chem. 61, 231-238 (2015).

23. Kullo, I. J. \& Bailey, K. R. Design of a controlled trial of cascade screening for hypercholesterolemia: the (cash) study. J. Pers. Med. 8, 27 (2018).

24. Haralambos, K. et al. Clinical experience of scoring criteria for Familial Hypercholesterolaemia (FH) genetic testing in Wales. Atherosclerosis 240, 190-196 (2015).

25. Shen, $H$. et al. Familial defective apolipoprotein B-100 and increased low-density lipoprotein cholesterol and coronary artery calcification in the old order amish. Arch. Intern. Med. 170, 1850-1855 (2010).

26. Grzymski, J. J. et al. Population genetic screening efficiently identifies carriers of autosomal dominant diseases. Nat. Med. 26, 1235-1239 (2020).

27. Klarin, D. et al. Genetics of blood lipids among $\sim 300,000$ multi-ethnic participants of the Million Veteran Program. Nat. Genet. 50, 1514-1523 (2018).

28. Olson, J. E. et al. The mayo clinic biobank: a building block for individualized medicine. Mayo Clin. Proc. 88, 952-962 (2013).

29. Kullo, I. J. et al. The Return of Actionable Variants Empirical (RAVE) Study, a mayo clinic genomic medicine implementation study: design and initial results. Mayo Clin. Proc. 93, 1600-1610 (2018).

30. Zouk, H. et al. Harmonizing clinical sequencing and interpretation for the eMERGE III network. Am. J. Hum. Genet. 105, 588-605 (2019).

31. Reid, J. G. et al. Launching genomics into the cloud: deployment of Mercury, a next generation sequence analysis pipeline. BMC Bioinformatics 15, 30 (2014).

32. Li, H. \& Durbin, R. Fast and accurate short read alignment with Burrows-Wheeler transform. Bioinformatics 25, 1754-1760 (2009).

33. Richards, S. et al. Standards and guidelines for the interpretation of sequence variants: a joint consensus recommendation of the American College of Medical Genetics and Genomics and the Association for Molecular Pathology. Genet. Med. 17, 405-424 (2015).

34. Li, Q. \& Wang, K. InterVar: clinical interpretation of genetic variants by the 2015 ACMG-AMP Guidelines. Am. J. Hum. Genet. 100, 267-280 (2017).

35. Karczewski, K. J. et al. The ExAC browser: displaying reference data information from over 60000 exomes. Nucleic Acids Res. 45, D840-D845 (2017).

36. Lek, M. et al. Analysis of protein-coding genetic variation in 60,706 humans. Nature 536, 285-291 (2016).

37. Landrum, M. J. et al. ClinVar: Public archive of relationships among sequence variation and human phenotype. Nucleic Acids Res 42, D980-D985 (2014).

38. Fokkema, I. F. A. C., Den Dunnen, J. T. \& Taschner, P. E. M. LOVD: Easy creation of a locus-specific sequence variation database using an 'LSDB-in-a-Box' approach. Hum. Mutat. 26, 63-68 (2005).

39. Stenson, P. D. et al. The human gene mutation database: 2008 update. Genome Med. 1, 13 (2009).

40. Safarova, M. S., Liu, H. \& Kullo, I. J. Rapid identification of familial hypercholesterolemia from electronic health records: The SEARCH study. J. Clin. Lipidol. 10, 1230-1239 (2016). 
41. Balder, J. W. et al. Lipid and lipoprotein reference values from 133,450 Dutch Lifelines participants: Age- and gender-specific baseline lipid values and percentiles. J. Clin. Lipidol. 11, 1055-1064 (2017).

\section{ACKNOWLEDGEMENTS}

We thank Ms. Luanne Wussow for help with preparation of the manuscript. This study was funded as part of the NHGRI-supported electronic MEdical Records and GEnomics (eMERGE) network (U01HG006379) and the Mayo Center for Individualized Medicine. The Mayo Clinic Biobank is funded by the Mayo Clinic Center for Individualized Medicine. Dr. Kullo is additionally funded by NHLBI grant K24HL137010. The content is solely the responsibility of the authors and does not necessarily represent the official views of the National Institutes of Health.

\section{AUTHOR CONTRIBUTIONS}

I.J.K. designed the study. M.J., D.C.K, M.S., X.F., L.A., O.D., and S.S. conducted the study and collected the data. I.J.K., J.E.O, D.C.K, and A.A.M coordinated the study. O.D., D.C.K, A.A.M, and S.S. performed the statistical analyses. S.S., M.J. and I.J.K wrote the manuscript and all authors read and approved the final manuscript. S.S. and M.J. are considered as co-first authors.

\section{COMPETING INTERESTS}

The authors declare no competing interests.

\section{ADDITIONAL INFORMATION}

Supplementary information The online version contains supplementary material available at https://doi.org/10.1038/s41525-021-00190-z.

Correspondence and requests for materials should be addressed to I.J.K.

Reprints and permission information is available at http://www.nature.com/ reprints

Publisher's note Springer Nature remains neutral with regard to jurisdictional claims in published maps and institutional affiliations.

Open Access This article is licensed under a Creative Commons Attribution 4.0 International License, which permits use, sharing, adaptation, distribution and reproduction in any medium or format, as long as you give appropriate credit to the original author(s) and the source, provide a link to the Creative Commons license, and indicate if changes were made. The images or other third party material in this article are included in the article's Creative Commons license, unless indicated otherwise in a credit line to the material. If material is not included in the article's Creative Commons license and your intended use is not permitted by statutory regulation or exceeds the permitted use, you will need to obtain permission directly from the copyright holder. To view a copy of this license, visit http://creativecommons. org/licenses/by/4.0/.

(c) The Author(s) 2021, corrected publication 2021 